Claremont Colleges

Scholarship@ Claremont

All HMC Faculty Publications and Research

HMC Faculty Scholarship

4-1-1989

\title{
$\Gamma$ to X Transport of Photoexcited Electrons in Type II GaAs/AlAs Multiple Quantum Well Structures
}

Peter N. Saeta

Harvey Mudd College

John F. Federici

AT\& $T$ Bell Laboratories

R. J. Fischer

AT\&T Bell Laboratories

Benjamin I. Greene

AT\&T Bell Laboratories

L. Pfeiffer

AT\&T Bell Laboratories

See next page for additional authors

\section{Recommended Citation}

" $\Gamma$ to $X$ transport of photoexcited electrons in type II GaAs/AlAs multiple quantum well structures," P. Saeta, J. F. Federici, R. J. Fischer, B. I. Greene, L. Pfeifer, R. C. Spitzer, and B. A. Wilson, Appl. Phys. Lett. 54, 1681 (1989). doi: 10.1063/1.101302

This Article is brought to you for free and open access by the HMC Faculty Scholarship at Scholarship @ Claremont. It has been accepted for inclusion in All HMC Faculty Publications and Research by an authorized administrator of Scholarship @ Claremont. For more information, please contact scholarship@cuc.claremont.edu. 
Authors

Peter N. Saeta, John F. Federici, R. J. Fischer, Benjamin I. Greene, L. Pfeiffer, R. C. Spitzer, and B. A. Wilson 


\section{AIP Applied Physics \\ Letters}

$\Gamma$ to $X$ transport of photoexcited electrons in type II GaAs/AIAs multiple quantum well structures

P. Saeta, J. F. Federici, R. J. Fischer, B. I. Greene, L. Pfeiffer et al.

Citation: Appl. Phys. Lett. 54, 1681 (1989); doi: 10.1063/1.101302

View online: http://dx.doi.org/10.1063/1.101302

View Table of Contents: http://apl.aip.org/resource/1/APPLAB/v54/i17

Published by the AIP Publishing LLC.

Additional information on Appl. Phys. Lett.

Journal Homepage: http://apl.aip.org/

Journal Information: http://apl.aip.org/about/about_the_journal

Top downloads: http://apl.aip.org/features/most_downloaded

Information for Authors: http://apl.aip.org/authors

\section{ADVERTISEMENT}
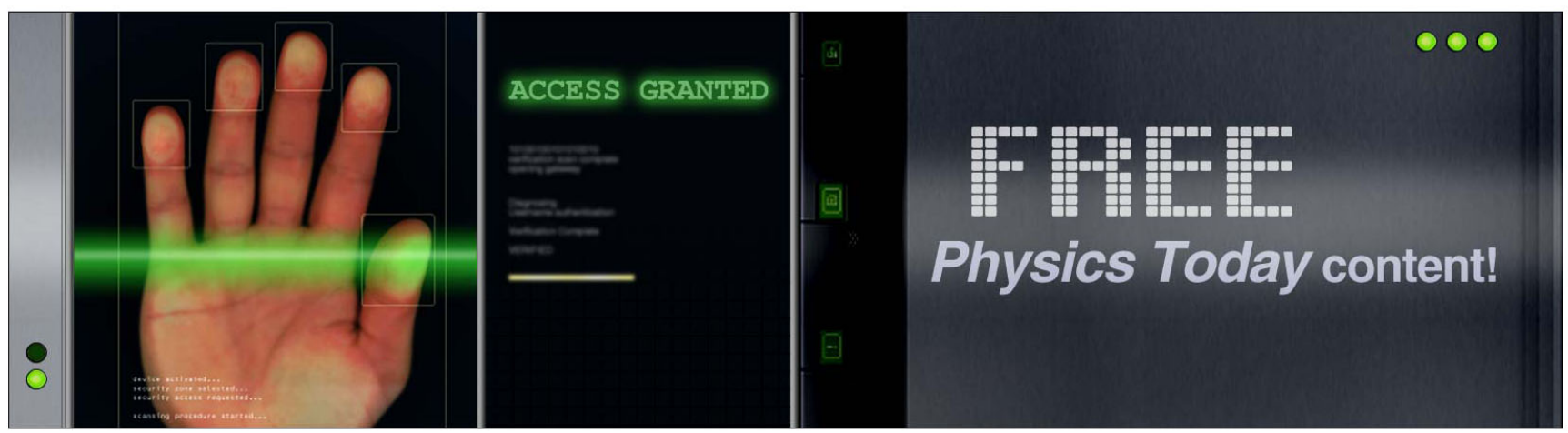


\title{
$\Gamma$ to $X$ transport of photoexcited electrons in type II GaAs/AlAs multiple quantum well structures
}

\author{
P. Saeta, ${ }^{\text {a) }}$ J. F. Federici, R. J. Fischer, B. I. Greene, L. Pfeiffer, A. C. Spitzer, \\ and B. A. Wilson ${ }^{\text {by }}$ \\ AT\&T Bell Laboratories, Murray Hill, New Jersey 07974
}

(Received 21 November 1988; accepted for publication 6 February 1989)

\begin{abstract}
We report novel femtosecond time-resolved measurements performed on staggered type II GaAs/AlAs multiple quantum well structures. Photoexcited electrons were determined to transfer from the $\Gamma$ valley of the GaAs layers to the $X$ valleys of the AlAs in 100 and 400 fs for 8- and 11-monolayer-thick GaAs samples, respectively.
\end{abstract}

For the past several years considerable attention has been focused on hot carrier dynamics in bulk semiconductors and multiple quantum well structures (MQWSs). Carrier-carrier and carrier-phonon interactions too rapid to be observed on a nanosecond or picosecond timescale have recently been directiy resolved with femtosecond light pulses. ${ }^{i-6}$ Numerous studies have now resulted in a reasonably clear picture of the evolution of photoexcited carriers in bulk GaAs as well as in conventional GaAs/GaAlAs MQWSs. ${ }^{-7}$

Advances in molecular beam epitaxy and other nanoengineering techniques have recently made available a variety of MQWSs and superlattices which have attracted attention not only for their inherent interest, but also for their potential use in optoelectronic devices. Excellent reviews on these structures have appeared in the literature. ${ }^{8,9}$ For the purposes of this letter, we discuss two kinds of MQWSs, commonly designated "type I" and "staggered type II." Brieffy, type I structures are those in which the highest lying valence band and the lowest lying conduction band (CB) occur in the same layer. This layer has the narrower band gap, and is usually referred to as the "well." The majority of time-resolved studies have been on type I MQWSs. Staggered type II structures are characterized as having staggered bands with the highest valence band occurring in one layer and the lowest conduction band in the other. In these structures, excited carriers physically segregate with one carrier remaining in the narrower-band-gap material and the others transferring to the lower energy states occurring in the adjacent layer. ${ }^{10}$ In the GaAs/AlAs system, by varying the GaAs well width, and hence the confinement energy of the $\Gamma$-derived $C B$ states within the GaAs layer, it is possible to tune the lowest lying conduction-band states in the GaAs above the $X$-derived states in the AlAs layers, thereby achieving a type II configuration.

The samples used for these experiments consisted of 50 periods of either 8 or 11 monolayers of GaAs alternating with 25 monolayers of AlAs, grown on a GaAs substrate separated by a $100 \AA$ AlAs etch stop layer. Samples were fixed, quantum well side down, to a fused silica disk, polished, and etched, leaving only the etch stop and quantum wells.

\footnotetext{
a) AT\&T Bell Laboratories, Ph.D. scholar, Harvard University, Cambridge, MA 02138.

b) Present address: Jet Propulsion Laboratory, Pasadena, CA 91125.
}

Spectroscopic characterization of type II GaAs/AlAs MQWSs is more readily achieved by iuminescence rather than by direct absorption, due to the forbidden nature of the lowest optical transition. ${ }^{11-14}$ Such luminescence spectra for the 11-monolayer sample, displayed in Fig. 1, clearly show the doubly forbidden (both in momentum and real space) $X \rightarrow \Gamma$ zero phonon transition at $\sim 1.72 \mathrm{eV}$ as well as the direct $\Gamma \rightarrow \Gamma$ transition at $\sim 1.9 \mathrm{eV}$. These data were obtained at $6 \mathrm{~K}$ with $2.5 \times 10^{-1} \mathrm{~W} / \mathrm{cm}^{2} 514 \mathrm{~nm}$ cw excitation for the indirect transition, and $2.5 \mathrm{~W} / \mathrm{cm}^{2}$ for the direct transition. The relative intensities of buminescence for the direct and indirect transitions have been observed to be dependent on excitation intensity and temperature ${ }^{18-14}$ Futhermore, we have observed that for our samples, as the excitation intensity is lowered to the detectability limit for direct luminescence, the relative yield of luminescence, indirect to direct, increased to roughly $10^{4}$.

Transient absorption spectroscopy was performed via a technique described in detail previously. ${ }^{15}$ Use of a dispersion-compensated white-light continuum probe pulse, and an amplified $10 \mathrm{~mm}$ portion of a continuum pulse at either $1.97 \mathrm{or} .77 \mathrm{eV}$ as the excitation pulse, resulted in a $200 \mathrm{fs}$ full width at half-maximum cross-correlation function. This indicates laser pulse widths of roughly 100 fs. Data were obtained with the excitation and probing pulses orthogonally polarized. Samples were positioned at Brewsters angle with respect to the probe beam to minimize Fabry-Perot fringes.

Room-temperature spectroscopic results for the 11

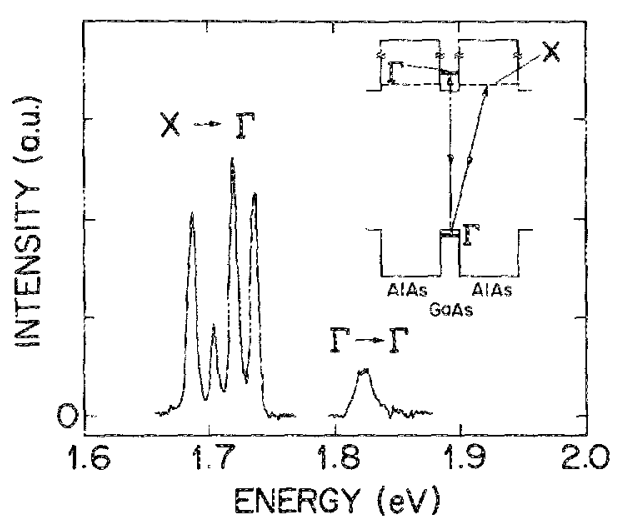

FIG. 1. cw luminescence spectra of the 1 monolayers GaAs/AlAs MQWS taken at $6 \mathrm{X}$. Excitation intersity for $\Gamma \rightarrow \Gamma$ spectrum was 10 times $X$ that for $X \rightarrow \Gamma$ spectrum. 


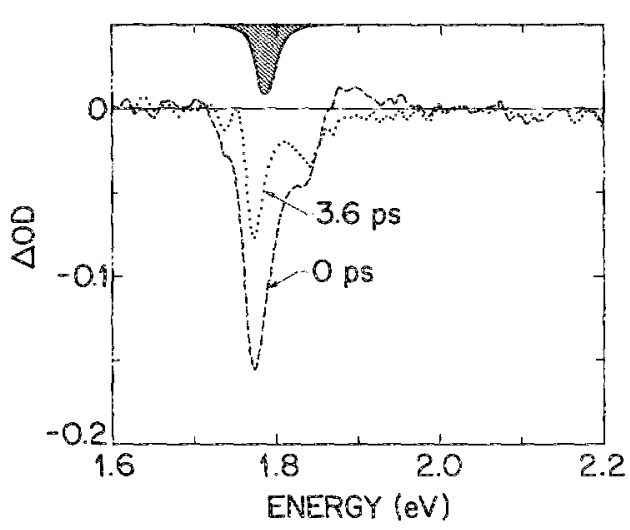

FIG. 2. Differential absorbance spectra of the 11 monolayers GaAs/AlAs MQWS taken at the temporal peak of bleaching (0 ps, dashcd line) and 3.6 ps later (3.6 ps, dotted line). Shaded curve indicates the spectrum of the excitation pulse.

monolayers sample are shown in Fig. 2. The data appear in the form of differential absorbance ( $\triangle O D$, optical density; $O D=-\log T$, where $T$ is the sample transmissivity). The spectrum of the excitation light appears shaded in on the top of the figure. The origin of the delay time was assigned to be at the temporal peak of the sample response. Such a temporal trace (obtained by monitoring sample transmission at the peak of its spectral response with a photodiode and a $10 \mathrm{~nm}$ full width at half-maximum interference filter centered at $700 \mathrm{~nm}$ ) appears in Fig. 3. Data in Fig. 2 were obtained with incident excitation intensity of roughly $1 \times 10^{9} \mathrm{~W} / \mathrm{cm}^{2}$ while data in Fig. 3 were obtained with excitation of roughly half this intensity. Similar spectral and kinetic results were obtained with the 8 monolayers sample. In this case, however, we observed a significantly faster relaxation time.

After the initial dramatic changes that occur by the first $\sim 3.0$ ps (see Figs. 2 and 3), essentially no further changes in the differential absorption spectrum were observed out to 30 ps, the longest delay time investigated. Figure 4 displays the 11 -monolayer sample absorbence spectrum as a function of delay time. The solid line (unexcited absorption spectrum) was obtained with a $\mathrm{cw}$ white-light source. The excited absorption spectra at 0 and 3.6 ps delay were obtained by adding the data in Fig. 2 to the unexcited absorbance spectrum.

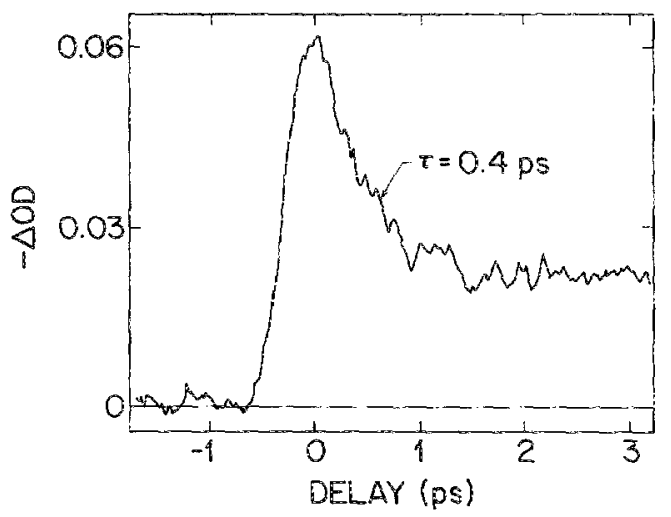

FiG. 3. Temporal trace of the 11 monolayers GaAs/AlAs MQWS sample optical response monitored at $700 \mathrm{~nm}(1.77 \mathrm{eV})$.
We estimate from our data characteristic material relaxation times of 100 and 400 fs for the 8 and 11 monolayers samples, respectively, and argue below that this is atributable to the transfer of photoexcited $\Gamma$ electrons to the $X$ valleys in the AlAs layers. Previous ferntosecond studies on type I GaAs MQWSs, cw luminescence data, and our own time-resolved work support this conclusion.

Insight into the ultrafast dymamics of these type II sys* tems can be gleaned from differential spectra (Fig. 2) taken at the peak of the bleaching and when the differential transmission assumes its persistent value (Fig. 3). The spectral width of the peak at $t=0$ is roughly three times the widh of the pump, and centered at slightly lower energy, evidence of ultrafast scattering of excited electrons out of initialiy occu. pied states. ${ }^{4}$ As is clear from the absorption spectrum (Fig. 4) at $t=0$, excitonic features have disappeared and carriers at the band edge have been largely depleted. By $\sim 3.0$ ps later, the dominant effect is partial recovery of the band edge absorption. Additional subtle but reproducible spectral changes appear at higher energies, up to $200 \mathrm{meV}$ above the band edge. Spectra taken at still Ionger delays (up to $30 \mathrm{ps}$ ) remain essentially unchanged in shape and magnitude.

Spectroscopic signatures of $\Gamma \rightarrow X$ scattering are expected to be both partial recovery of the initially induced bleaching and an electric field induced modification of the band edge absorption. Absorption recovery is attributable to deoccupation of the initialiy populated $\Gamma$ electron states via $\Gamma \rightarrow X$ scattering, whereas physical charge separation is expected to produce an electric field. The magnitude and spatial extent of this field would depend on the detailed nature of the relaxed charge distribution. The effect that such a field would have on the absorption spectrum is a measure of the perturbation it creates on the electron and hole energy levels in the GaAs layers.

Observed efficiencies for $\Gamma \rightarrow \Gamma$ luminescence of $\sim 10^{-4}$ rule out the possibility that the persistent bleaching observed in our data is due to $\Gamma$ electrons in the GaAs layers. We attribute this bleaching instead to the presence of GaAs holes unable to recombine with electrons bottlenecked in the

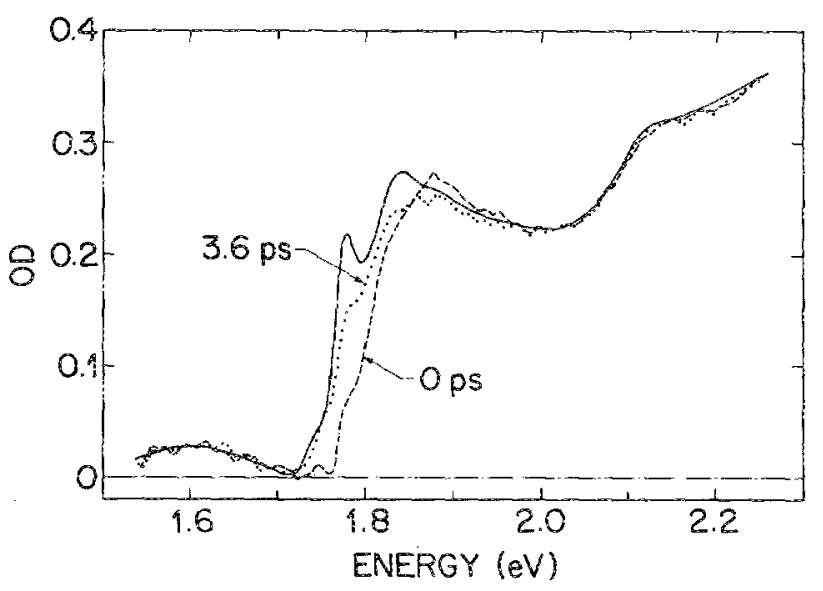

FIG. 4. Absorbance spectra of the 11 monolayers GaAs/AlAs MQWS sample without photoexcitation (solid line), at the temporal peak of the optical response ( $0 \mathrm{ps}$, dashed line), and after relaxation is complete ( $3.6 \mathrm{ps}$, dotted line). 
AlAs. The observed subpicosecond decay is necessarily assigned to the transport of $\Gamma$ GaAs electrons to the adjacent AlAs layers. Measured relative yields of luminescence, indirect or direct, are consistent with a $\sim 100$ fs $\Gamma \rightarrow X$ relaxation. Intensity-dependent relative yields are understood via a model that stipulates a relatively low density of indirect luminescence centers which can be saturated. Thermally activated nonradiative relaxation pathways also compete with $X \rightarrow \Gamma$ indirect luminescence. In the absence of competitive nonradiative pathways, i.e., at low intensity and low temperature, a luminescence ratio of $10^{4}$, indirect to direct, puts an effective upper limit on the $\Gamma$ electron lifetime (assuming a 1 ns natural radiative lifetime in GaAs) of $100 \mathrm{fs}$.

$\Gamma \rightarrow X$ relaxation in type II MQWSs requires electron transport from, in our case, a GaAs layer to an adjacent AlAs layer. Obvious!y, this cannot occur any faster than the $z$ component ( 1 to the layers) of the electron velocity would allow. With a sample GaAs layer thickness of $\sim 25-30 \AA$, and a calculated Fermi velocity (corresponding to an estimated conduction electron density of $2 \times 10^{18} / \mathrm{cm}^{3}$ ) of $\sim 5 \times 10^{6} \mathrm{~cm} / \mathrm{s}$, a $\Gamma \rightarrow X$ relaxation on the order of $100 \mathrm{fs}$ appears quite reasonable. This assumes efficient momentum transfer $p_{x}, p_{y} \rightarrow p_{z}$ and is suggested only as an upper limit for the rate of electron transport.

Identification of spectral manifestations of internal electric field effects is complicated by the presence of persistent band edge bleaching. Beyond this bleaching effect, there is no obvious evidence of a dramatically shifted or broadened band edge subsequent to initial relaxation.

We point out that the modifed potentials associated with externally applied electric fields [as in the quantum confined Stark effect ${ }^{16}$ (QCSE)] are qualitatively different from the modifed potentials resulting from charge separation in type II MQWSs. Whereas the external feld leading to the QCSE uniformly skews the potential, the internal electric field in the type II structures produces a pericdic distor. tion in the potential. Rough estimates for GaAs well thicknesses comparable to our samples indiate energy shifts of $\sim 5$ meV, too small to be experimentally resolved.

In conclusion, we have presented time-resolved optical absorption measurements performed on a type II GaAs/
AlAs MQWS. The data indicate an ultrafast (subpicosecond) relaxation pathway, which we attribute to $\Gamma \rightarrow X$ carrier scattering. Electron transport from the GaAs layers to the AIAs layers is, therefore, determined to occur on this timescale.

The authors wish to thank N. A. Olsson, Kuochou Tai, and R. A. Logan for their expert help with sample preparation, and Carl E. Bonner for assistance with luminescence spectroscopy. We wish to also thank J. Orenstein, S. Schmitt-Rink, M.-H. Meynadier, and W. Wilson for numerous stimulating discussions regarding this work. P. Saeta gratefuly acknowledges AT\&T Bell Laboratories for his support as a Ph. D. scholar.

'C. V. Shank, R. L. Fork, R. F. Lelteny, and Jagdeep Shah, Phys. Rev. Lett. 42, $112(1979)$.

${ }^{2}$ C. V. Shank, R. L. Fork, R. Yen, J. Srah, B. I. Greene, A. C. Gossard, and C. Weisbuch, Solid State Commun. 87,981 (1983).

${ }^{3} J$. L. Oudar, D. Hukin, A. Migus, A. Antonetti, and F. Alexandre, Phys. Rev. Lett. 55, 2074 (1985).

${ }^{4}$ W. H. Knox, C. Hirlimann, D. A. B. Miller, J. Shah, D. S. Chemla, and C. V. Shank, Phys. Rev. Lett. 56, 1191 (1986).

${ }^{5}$ W. Z. Lin, L. G. Fujimoto, E. P. Ippen, and R. A. Logan, Appl. Phys. Lett. 58, 124 (1987).

"W. H. Knox, D. S. Chemla, G. Livescu, J. Cunningham, and J. E. Henry, Phys. Rev. Lett. 61, 1290 (1988).

${ }^{7}$ J. Shah, IEEE J. Quantum Electron. QH-22, 1728 (1986).

${ }^{8}$ L. Esaki, IEEE J. Quantum Electron. QE-22, 1611 (1986).

${ }^{2} \mathrm{C}$. Weisbuch, in Semiconductors and Semimetals, edited by R. Dingle (Acadcmic, San Diego, 1987), Vol. 24.

${ }^{10}$ B. A. Wilson, IEEE J. Quantum Electron. 24, 1763 (1988).

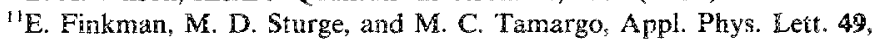
1299 (1986).

"G. Danan, B. Etienne, F. Mollot, R. Planel, A. M. Jean-Louis, F. Alexandre, B. Jusserand, G. LeRoux, J. Y. Marzin, H. Savary, and B. Sermage, Phys. Rev. B 35, 6207 (1987).

${ }^{13}$ F. Minami, K. Hirate, K. Era, F. Yao, and Y. Masumoto, Phys. Rev. B $36,2875(1987)$.

${ }^{14}$ P. Dawson, K. J. Moore, and C. T. Foxton, Proc. Soe. Thoto-Opt. Instrum. Eng. 792, 208 (1987).

${ }^{15}$ B. I. Greene, J. F. Mueller, J. Orenstein, D. H. Rapkine, S. Schmitt-Rink, and M. Thakur, Phys. Rev. Lett. 61, 325 (1988).

'D. A. B. Miller, D. S. Chemla, T. C. Damen, A. C. Gossard, W. Wegmann, T. H. Wood, and C. A. Burrus, Phys. Rev. B 32, 1043 (1985). 